\title{
Note on a Casimir Energy Calculation for a Purely Dielectric Cylinder by Mode Summation
}

\author{
August Romeo and Kimball A. Milton $\ddagger$ \\ Oklahoma Center for High Energy Physics and Homer L. Dodge Department of \\ Physics and Astronomy, University of Oklahoma, Norman, OK 73019, USA
}

\begin{abstract}
We comment on a recent calculation of the zero-point energy for a dilute and infinitely long cylinder of purely-dielectric material. The vanishing result predicted by integration of van der Waals potentials is obtained.

PACS numbers: 42.50.Pq, 42.50.Lc, 11.10.Gh, 03.50.De
\end{abstract}

The Casimir effect is a change in the electromagnetic vacuum fluctuations brought about by the presence of boundaries. Particularly, cylindrical surfaces limiting dielectric media were considered in [1]. One of the first versions of that paper inspired an unpublished calculation, by Romeo, of the van der Waals energy for a purely dielectric cylinder in the dilute-dielectric approximation, which yielded a null result. That calculation found a tribune in appendix B of the final version of [1] and, eventually, unpublished work by Milonni and ref.[2] by Barton provided independent confirmations.

This finding aroused curiosity about the corresponding Casimir energy, which would have to show the predicted equality between both quantities [3] and, therefore, was expected to vanish similarly. The divergences of this problem were studied through its heat kernel coefficents in [4], and the expected vanishing was first verified in [5], where the Casimir pressure was obtained from the expectation value of the stress-energy tensor using Green's functions. Next, a calculation of the Casimir energy based on the mode summation method [6] was completed. The present note offers a comment on that work.

Let $J_{m}, H_{m}$ denote the Bessel and Hankel functions (for $y>0, H_{m}(y) \equiv H_{m}^{(1)}(y)$ ). Given an infinitely long cylinder of radius $a$, oriented along the $z$-axis, with permittivity and permeability $\left(\varepsilon_{1}, \mu_{1}\right)$, surrounded by a medium with permittivity and permeability $\left(\varepsilon_{2}, \mu_{2}\right)$, the eigenfrequencies $\omega$ of the Maxwell equations with the adequate boundary $\ddagger$ on sabbatical leave at the Department of Physics, Washington University, St. Louis, MO 63130 USA 
conditions are the solutions of:

$$
\begin{gathered}
f_{m}\left(k_{z}, \omega\right)=0, \quad m \in \mathbb{Z}, \quad k_{z} \in \mathbb{R} \\
f_{m}\left(k_{z}, \omega\right) \equiv \frac{1}{\Delta^{2}}\left[\Delta_{m}^{\mathrm{TE}}(x, y) \Delta_{m}^{\mathrm{TM}}(x, y)-m^{2} \frac{a^{4} \omega^{2} k_{z}^{2}}{x^{2} y^{2}}\left(\varepsilon_{1} \mu_{1}-\varepsilon_{2} \mu_{2}\right)^{2} J_{m}^{2}(x) H_{m}^{2}(y)\right]
\end{gathered}
$$

(see $[7,1])$, where

$$
\begin{gathered}
\Delta=-\frac{2 i}{\pi} \\
\Delta_{m}^{\mathrm{TE}}(x, y)=\mu_{1} y J_{m}^{\prime}(x) H_{m}(y)-\mu_{2} x J_{m}(x) H_{m}^{\prime}(y), \\
\Delta_{m}^{\mathrm{TM}}(x, y)=\varepsilon_{1} y J_{m}^{\prime}(x) H_{m}(y)-\varepsilon_{2} x J_{m}(x) H_{m}^{\prime}(y), \\
x=\lambda_{1} a, \quad y=\lambda_{2} a, \quad \lambda_{i}^{2}=\varepsilon_{i} \mu_{i} \omega^{2}-k_{z}, \quad i=1,2 .
\end{gathered}
$$

The $m$ index is the azimuthal quantum number, $k_{z}$ is the momentum along the cylinder axis, and $p$ labels the zeroes of $f_{m}\left(k_{z}, \omega\right)$. In fact $f_{m}=-\Delta^{-2} \Xi$, being $\Xi$ the same object as in [5] and $\Delta^{-2}$ a factor introduced for convenience. The velocities of light in each media are $c_{i}=\left(\varepsilon_{i} \mu_{i}\right)^{-1 / 2}, i=1,2$.

If medium 1 is purely dielectric and medium 2 is vacuum, $\varepsilon_{1}=\varepsilon, \mu_{1}=1$, $\varepsilon_{2}=\mu_{2}=1$ (obviously, $c_{2}=1$ ). Further,

$$
\omega=a^{-1}\left(y^{2}+\widehat{k}^{2}\right)^{1 / 2}, \quad x^{2}=y^{2}+(\varepsilon-1)\left(y^{2}+\widehat{k}^{2}\right), \quad \widehat{k} \equiv k_{z} a .
$$

The Casimir energy per unit length stems from the mode sum

$$
\mathcal{E}_{C}=\frac{1}{2} \hbar \int_{-\infty}^{\infty} \frac{d k_{z}}{2 \pi} \sum_{m} \sum_{p} \omega_{m, p, k_{z}}
$$

which is divergent, and will be regularized appropriately (see below). Reference [4] tells us that, up through the order of $(\varepsilon-1)^{2}$, there are no ambiguities, because the heat kernel coefficient which would multiply them is of $\mathcal{O}\left((\varepsilon-1)^{3}\right)$. Thus, we may just set

$\mathcal{E}_{C}(s)=\frac{\hbar}{2} \int_{-\infty}^{\infty} \frac{d k_{z}}{2 \pi} \sum_{m} \sum_{p} \omega_{m, p, k_{z}}^{-s}=\frac{\hbar}{2} a^{s-1} \int_{-\infty}^{\infty} \frac{d \widehat{k}}{2 \pi} \sum_{m} \sum_{p}\left(y_{m, p}^{2}+\widehat{k}^{2}\right)^{-s / 2}$,

without any additional mass scale. $\mathcal{E}_{C}(s)$ is a function of the complex variable $s$, and our idea is to redefine (4) by analytic continuation of this function to $s=-1$, i.e.,

$$
\mathcal{E}_{C}=\lim _{s \rightarrow-1} \mathcal{E}_{C}(s)
$$

Once that $\widehat{k}, m$ have specific values, the sum over $p$ is expressed as a contour integral in complex $y$ plane:

$$
\mathcal{E}_{C}(s)=\frac{\hbar}{2} a^{s-1} \int_{-\infty}^{\infty} \frac{d \widehat{k}}{2 \pi} \sum_{m=-\infty}^{\infty} \frac{s}{2 \pi i} \int_{C} d y y\left(y^{2}+\widehat{k}^{2}\right)^{-s / 2-1} \ln f_{m},
$$

where $C$ is a circuit enclosing all the $y$ values corresponding to the positive zeroes of $f_{m}$ (the argument principle [8] derived from the residue theorem). When applying this method, one sometimes finds an asymptotic form $f_{m \text {,as }}$ of $f_{m}$ and then subtracts $\ln f_{m \text {,as }}$ from $\ln f_{m}$ in the integrand. In fact, the factors introduced in (1) relative to the original 
$f_{m}$ of [1] have the same effect as having divided that function by the leading part of $f_{m \text {,as }}$.

At this point, the logarithm function of $(7)$ is expanded in powers of $(\varepsilon-1)$, taking $y$ as an independent variable and $x$ as a function of $y, \widehat{k}, \varepsilon($ see $(3))$. Then,

$$
\begin{aligned}
\ln f_{m}= & {\left[L_{m 1}^{0}(y)+L_{m 1}^{1}(y)\left(y^{2}+\widehat{k}^{2}\right)\right](\varepsilon-1) } \\
& +\left[L_{m 2}^{00}(y)+L_{m 2}^{10}(y)\left(y^{2}+\widehat{k}^{2}\right)+L_{m 2}^{20}(y)\left(y^{2}+\widehat{k}^{2}\right)^{2}\right. \\
& \left.+L_{m 2}^{11}(y)\left(y^{2}+\widehat{k}^{2}\right) \widehat{k}^{2}\right](\varepsilon-1)^{2} \\
& +\mathcal{O}\left((\varepsilon-1)^{3}\right),
\end{aligned}
$$

where

$$
\begin{aligned}
& L_{m 1}^{0}(y)=\frac{1}{4} y J_{m}^{\prime}(y) H_{m}(y), \\
& L_{m 1}^{1}(y)=\frac{1}{\Delta y} \Delta_{m}^{(1,0)}(y), \\
& L_{m 2}^{00}(y)=-\frac{1}{2 \Delta^{2}} y^{2} J_{m}^{\prime 2}(y) H_{m}^{2}(y), \\
& L_{m 2}^{10}(y)=-\frac{1}{2 \Delta^{2}}\left[\Delta_{m}^{(1,0)}(y) J_{m}^{\prime}(y) H_{m}(y)\right. \\
& \left.+\frac{\Delta}{y}\left(J_{m}^{\prime}(y)+y\left(1-\frac{m^{2}}{y^{2}}\right) J_{m}(y)\right) H_{m}(y)\right], \\
& L_{m 2}^{20}(y)=L_{m 2}^{20 A}(y)+L_{m 2}^{20 B}(y), \quad\left\{\begin{aligned}
L_{m 2}^{20 A}(y) & =\frac{1}{4 \Delta y^{2}}\left(\Delta_{m}^{(2,0)}(y)-\frac{1}{y} \Delta_{m}^{(1,0)}(y)\right), \\
L_{m 2}^{20 B}(y) & =-\frac{1}{4 \Delta^{2} y^{2}}\left(\Delta_{m}^{(1,0)}(y)\right)^{2},
\end{aligned}\right. \\
& L_{m 2}^{11}(y)=-\frac{m^{2}}{\Delta^{2} y^{4}} J_{m}^{2}(y) H_{m}^{2}(y),
\end{aligned}
$$

with

$$
\begin{aligned}
& \Delta_{m}^{(1,0)}(y)=-\frac{1}{y}\left[y^{2} J_{m}^{\prime}(y) H_{m}^{\prime}(y)+\left(y^{2}-m^{2}\right) J_{m}(y) H_{m}(y)\right]-\left(J_{m}(y) H_{m}(y)\right)^{\prime} \\
& \Delta_{m}^{(2,0)}(y)=\left(\Delta_{m}^{(1,0)}(y)\right)^{\prime}-\left(1-\frac{m^{2}+1}{y^{2}}\right) \Delta, \quad\left(\Delta_{m}^{(1,0)}(y)\right)^{\prime} \equiv \frac{d}{d y} \Delta_{m}^{(1,0)}(y) .
\end{aligned}
$$

Now, (8) is inserted into (7). The obtained expression involves integrals of the form

$$
I \equiv \int_{-\infty}^{\infty} d \widehat{k} \int_{C} d y y F(y)\left(y^{2}+\widehat{k}^{2}\right)^{-\alpha} \widehat{k}^{2 \beta}
$$

where $C$ is the contour of $(7)$ and $F$ satisfies $F(-i v)=F(i v)$ for $v \in \mathbb{R}$, as well as having good asymptotic properties (the role of $F$ is played by the $L_{m}$ 's of $(9),(10)$ ). Examining the $\left(y^{2}+\widehat{k}^{2}\right)$ powers in (7), (8), one sees that, in the required cases, $\alpha=s / 2+1, s / 2, s / 2-1$, and $\beta=0$ except for one integral with $\beta=1$. Analytic continuation in $s$ obviously amounts to analytic continuation in $\alpha$. Following [6], the value of $I$ is given by

$$
I=-2 i \mathrm{~B}\left(\beta+\frac{1}{2}, 1-\alpha\right) \sin (\pi \alpha) \int_{0}^{\infty} d v v^{2-2 \alpha+2 \beta} F(i v),
$$


where $\mathrm{B}$ denotes the Euler beta function (about the mathematical basis, see also $[9,10]$ ). Note that for $s=-1$, i.e., $\alpha=1 / 2,-1 / 2,-3 / 2$, and for $\beta=0,1$, the beta and sine functions are finite. Application of formula (12) to Eqs. (7), (8) gives:

$$
\mathcal{E}_{C}(s)=\mathcal{E}_{C 1}(s)(\varepsilon-1)+\mathcal{E}_{C 2}(s)(\varepsilon-1)^{2}+\mathcal{O}\left((\varepsilon-1)^{3}\right),
$$

where

$$
\left\{\begin{array}{c}
\mathcal{E}_{C 1}(s)=\mathcal{E}_{C 1}^{0}(s)+\mathcal{E}_{C 1}^{1}(s) \\
\mathcal{E}_{C 1}^{0}(s)=-\frac{\hbar}{2} \frac{s a^{s-1}}{2 \pi^{2}} \mathrm{~B}\left(\frac{1}{2},-\frac{s}{2}\right) \sin \left(-\pi \frac{s}{2}\right) \sum_{m=-\infty}^{\infty} \int_{0}^{\infty} d v v^{-s} L_{m 1}^{0}(i v), \\
\mathcal{E}_{C 1}^{1}(s)=-\frac{\hbar}{2} \frac{s a^{s-1}}{2 \pi^{2}} \mathrm{~B}\left(\frac{1}{2}, 1-\frac{s}{2}\right) \sin \left(\pi \frac{s}{2}\right) \sum_{m=-\infty}^{\infty} \int_{0}^{\infty} d v v^{2-s} L_{m 1}^{1}(i v),
\end{array}\right.
$$

and

$$
\left\{\begin{aligned}
\mathcal{E}_{C 2}(s)=\mathcal{E}_{C 2}^{00}(s)+\mathcal{E}_{C 2}^{10}(s)+\mathcal{E}_{C 2}^{20 A}(s)+\mathcal{E}_{C 2}^{20 B}(s)+\mathcal{E}_{C 2}^{11}(s) \\
\mathcal{E}_{C 2}^{00}(s)=-\frac{\hbar}{2} \frac{s a^{s-1}}{2 \pi^{2}} \mathrm{~B}\left(\frac{1}{2},-\frac{s}{2}\right) \sin \left(-\pi \frac{s}{2}\right) \sum_{m=-\infty}^{\infty} \int_{0}^{\infty} d v v^{-s} L_{m 2}^{00}(i v) \\
\mathcal{E}_{C 2}^{10}(s)=-\frac{\hbar}{2} \frac{s a^{s-1}}{2 \pi^{2}} \mathrm{~B}\left(\frac{1}{2}, 1-\frac{s}{2}\right) \sin \left(\pi \frac{s}{2}\right) \sum_{m=-\infty}^{\infty} \int_{0}^{\infty} d v v^{2-s} L_{m 2}^{10}(i v), \\
\mathcal{E}_{C 2}^{20 A, B}(s)=-\frac{\hbar}{2} \frac{s a^{s-1}}{2 \pi^{2}} \mathrm{~B}\left(\frac{1}{2}, 2-\frac{s}{2}\right) \sin \left(-\pi \frac{s}{2}\right) \sum_{m=-\infty}^{\infty} \int_{0}^{\infty} d v v^{4-s} L_{m 2}^{20 A, B}(i v), \\
\mathcal{E}_{C 2}^{11}(s)=-\frac{\hbar}{2} \frac{s a^{s-1}}{2 \pi^{2}} \mathrm{~B}\left(\frac{3}{2}, 1-\frac{s}{2}\right) \sin \left(\pi \frac{s}{2}\right) \sum_{m=-\infty}^{\infty} \int_{0}^{\infty} d v v^{4-s} L_{m 2}^{11}(i v) .
\end{aligned}\right.
$$

With $\mathcal{E}_{C 1}^{0}(s)$ taken from $(14)$, and $L_{m 1}^{0}(i v)$ from (9), we arrive at

$\mathcal{E}_{C 1}^{0}(s)=-\frac{\hbar}{2} \frac{s a^{s-1}}{2 \pi^{2}} \mathrm{~B}\left(\frac{1}{2},-\frac{s}{2}\right) \sin \left(-\pi \frac{s}{2}\right) \sum_{m=-\infty}^{\infty} \int_{0}^{\infty} d v v^{1-s} I_{m}^{\prime}(v) K_{m}(v)$.

The beta and sine functions are already finite at $s=-1$, and the integral will be reexpressed by introducing the factor $1=-v W\left[I_{m}(v), K_{m}(v)\right]=-v\left[I_{m}(v) K_{m}^{\prime}(v)-\right.$ $\left.I_{m}^{\prime}(v) K_{m}(v)\right]$ for every $m$ :

$$
\begin{gathered}
\int_{0}^{\infty} d v v^{1-s} \sum_{m=-\infty}^{\infty} I_{m}^{\prime}(v) K_{m}(v)= \\
-\int_{0}^{\infty} d v v^{2-s} \sum_{m=-\infty}^{\infty} I_{m}(v) I_{m}^{\prime}(v) K_{m}(v) K_{m}^{\prime}(v)+\int_{0}^{\infty} d v v^{2-s} \sum_{m=-\infty}^{\infty} I_{m}^{\prime 2}(v) K_{m}^{2}(v) .
\end{gathered}
$$

The summations over $m$ will be performed by taking advantage of the addition theorem for the modified Bessel functions:

$$
\begin{aligned}
\sum_{m=-\infty}^{\infty} I_{m}(k r) K_{m}(k \rho) e^{i m \phi} & =K_{0}(k R(r, \rho, \phi)) \\
R(r, \rho, \phi) & =\sqrt{r^{2}+\rho^{2}-2 r \rho \cos \phi}, \quad \rho>r .
\end{aligned}
$$


Casimir Energy for a Purely Dielectric Cylinder by Mode Summation

Suitable manipulations of this identity $([11,12,5,6])$ yield:

$$
\begin{aligned}
& \int_{0}^{\infty} d v v^{2-s} \sum_{m=-\infty}^{\infty} I_{m}^{\prime 2}(v) K_{m}^{2}(v) \quad= \\
& \int_{0}^{\infty} d v v^{2-s} \sum_{m=-\infty}^{\infty} K_{m}^{\prime 2}(v) I_{m}^{2}(v) \quad= \\
& \int_{0}^{\infty} d v v^{2-s} \sum_{m=-\infty}^{\infty} I_{m}(v) I_{m}^{\prime}(v) K_{m}(v) K_{m}^{\prime}(v) \quad=\frac{1}{8 \pi^{1 / 2}} \frac{\Gamma\left(\frac{5-s}{2}\right) \Gamma^{2}\left(\frac{3-s}{2}\right) \Gamma\left(\frac{1-s}{2}\right)}{\Gamma(3-s)} \frac{\Gamma\left(\frac{s}{2}\right)}{\Gamma\left(\frac{s+1}{2}\right)} \\
& \int_{0}^{\infty} d v v^{2-s} \sum_{m=-\infty}^{\infty} m^{2} I_{m}(v) I_{m}^{\prime}(v) K_{m}(v) K_{m}^{\prime}(v)=\frac{1}{16 \pi^{1 / 2}} \frac{\Gamma^{4}\left(\frac{5-s}{2}\right)}{\Gamma(5-s)} \frac{\Gamma\left(\frac{s-2}{2}\right)}{\Gamma\left(\frac{s+1}{2}\right)} \\
& \int_{0}^{\infty} d v v^{4-s} \sum_{m=-\infty}^{\infty} I_{m}^{\prime 2}(v) K_{m}^{\prime 2}(v) \quad=\frac{1}{8 \pi^{1 / 2}}\left[\frac{\Gamma^{4}\left(\frac{5-s}{2}\right)}{\Gamma(5-s)} \frac{\Gamma\left(\frac{s}{2}\right)}{\Gamma\left(\frac{s+1}{2}\right)}\right. \\
& +\frac{\Gamma^{2}\left(\frac{5-s}{2}\right) \Gamma^{2}\left(\frac{3-s}{2}\right)}{\Gamma(4-s)} \frac{\Gamma\left(\frac{s-2}{2}\right)}{\Gamma\left(\frac{s-1}{2}\right)} \\
& \left.+\frac{1}{4} \frac{\Gamma\left(\frac{5-s}{2}\right) \Gamma^{2}\left(\frac{3-s}{2}\right) \Gamma\left(\frac{1-s}{2}\right)}{\Gamma(3-s)} \frac{\Gamma\left(\frac{s-4}{2}\right)}{\Gamma\left(\frac{s-3}{2}\right)}\right] \\
& \int_{0}^{\infty} d v v^{4-s} \sum_{m=-\infty}^{\infty} I_{m}^{2}(v) K_{m}^{2}(v) \\
& =\frac{1}{8 \pi^{1 / 2}} \frac{\Gamma^{4}\left(\frac{5-s}{2}\right)}{\Gamma(5-s)} \frac{\Gamma\left(\frac{s-4}{2}\right)}{\Gamma\left(\frac{s-3}{2}\right)} \\
& \int_{0}^{\infty} d v v^{2-s} \sum_{m=-\infty}^{\infty} m^{2} I_{m}^{2}(v) K_{m}^{2}(v) \quad=\frac{1}{16 \pi^{1 / 2}} \frac{\Gamma\left(\frac{7-s}{2}\right) \Gamma^{2}\left(\frac{5-s}{2}\right) \Gamma\left(\frac{3-s}{2}\right)}{\Gamma(5-s)} \frac{\Gamma\left(\frac{s-4}{2}\right)}{\Gamma\left(\frac{s-1}{2}\right)} \\
& \int_{0}^{\infty} d v v^{-s} \sum_{m=-\infty}^{\infty} m^{4} I_{m}^{2}(v) K_{m}^{2}(v) \\
& =\frac{1}{8 \pi^{1 / 2}}\left[\frac{3}{4} \frac{\Gamma^{4}\left(\frac{5-s}{2}\right)}{\Gamma(5-s)} \frac{\Gamma\left(\frac{s-4}{2}\right)}{\Gamma\left(\frac{s+1}{2}\right)}\right. \\
& +\frac{1}{2} \frac{\Gamma^{2}\left(\frac{5-s}{2}\right) \Gamma^{2}\left(\frac{3-s}{2}\right)}{\Gamma(4-s)} \frac{\Gamma\left(\frac{s-4}{2}\right)}{\Gamma\left(\frac{s-1}{2}\right)} \\
& \left.+\frac{1}{4} \frac{\Gamma\left(\frac{5-s}{2}\right) \Gamma^{2}\left(\frac{3-s}{2}\right) \Gamma\left(\frac{1-s}{2}\right)}{\Gamma(3-s)} \frac{\Gamma\left(\frac{s-4}{2}\right)}{\Gamma\left(\frac{s-3}{2}\right)}\right] \\
& \int_{0}^{\infty} d v v^{3-s} \sum_{m=-\infty}^{\infty} I_{m}^{\prime 2}(v) K_{m}(v) K_{m}^{\prime}(v) \quad= \\
& \int_{0}^{\infty} d v v^{3-s} \sum_{m=-\infty}^{\infty} K_{m}^{\prime 2}(v) I_{m}(v) I_{m}^{\prime}(v) \\
& =-\frac{1}{8 \pi^{1 / 2}}\left[\frac{\Gamma^{2}\left(\frac{5-s}{2}\right) \Gamma^{2}\left(\frac{3-s}{2}\right)}{\Gamma(4-s)} \frac{\Gamma\left(\frac{s}{2}\right)}{\Gamma\left(\frac{s+1}{2}\right)}\right. \\
& \left.+\frac{1}{2} \frac{\Gamma\left(\frac{5-s}{2}\right) \Gamma^{2}\left(\frac{3-s}{2}\right) \Gamma\left(\frac{1-s}{2}\right)}{\Gamma(3-s)} \frac{\Gamma\left(\frac{s-2}{2}\right)}{\Gamma\left(\frac{s-1}{2}\right)}\right] \\
& \int_{0}^{\infty} d v v^{3-s} \sum_{m=-\infty}^{\infty} I_{m}^{2}(v) K_{m}(v) K_{m}^{\prime}(v) \quad= \\
& \int_{0}^{\infty} d v v^{3-s} \sum_{m=-\infty}^{\infty} K_{m}^{2}(v) I_{m}(v) I_{m}^{\prime}(v) \quad=-\frac{1}{8 \pi^{1 / 2}} \frac{\Gamma^{2}\left(\frac{5-s}{2}\right) \Gamma^{2}\left(\frac{3-s}{2}\right)}{\Gamma(4-s)} \frac{\Gamma\left(\frac{s-2}{2}\right)}{\Gamma\left(\frac{s-1}{2}\right)} \\
& \int_{0}^{\infty} d v v^{1-s} \sum_{m=-\infty}^{\infty} m^{2} I_{m}^{2}(v) K_{m}(v) K_{m}^{\prime}(v)= \\
& \int_{0}^{\infty} d v v^{1-s} \sum_{m=-\infty}^{\infty} m^{2} K_{m}^{2}(v) I_{m}(v) I_{m}^{\prime}(v) \quad=-\frac{1}{16 \pi^{1 / 2}} \frac{\Gamma^{2}\left(\frac{5-s}{2}\right) \Gamma^{2}\left(\frac{3-s}{2}\right)}{\Gamma(4-s)} \frac{\Gamma\left(\frac{s-2}{2}\right)}{\Gamma\left(\frac{s+1}{2}\right)} .
\end{aligned}
$$

Although the left hand side of each integral is not initially defined for $s=-1$, the right hand side together with the remaining $s$ dependent factors in $\mathcal{E}_{C}(s)$ will eventually provide the desired extension to negative $s$ through the existing analytic continuations of the involved functions. Then, the poles at $s=-1,-3,-5, \ldots$ in the last dividing 
gamma functions, will give rise to zeros at these points.

Going back to $\mathcal{E}_{C 1}^{0}(s)$, since (19) show that the two integrals in the second line of (17) have the same value,

$$
\mathcal{E}_{C 1}^{0}(s)=0,
$$

even before setting $s=-1$.

Formulas (14) tell us that $\mathcal{E}_{C 1}^{1}(s)$ involves the integration of the $L_{m 1}^{1}(i v)$ function, defined by (9), (10). Therefore,

$$
\begin{gathered}
\mathcal{E}_{C 1}^{1}(s)=-\frac{\hbar}{2} \frac{s a^{s-1}}{2 \pi^{2}} \mathrm{~B}\left(\frac{1}{2}, 1-\frac{s}{2}\right) \sin \left(\pi \frac{s}{2}\right) \\
\times \sum_{m=-\infty}^{\infty} \int_{0}^{\infty} d v v^{2-s}\left[I_{m}^{\prime}(v) K_{m}^{\prime}(v)-\left(1+\frac{m^{2}}{v^{2}}\right) I_{m}(v) K_{m}(v)+\frac{1}{v}\left(I_{m}(v) K_{m}(v)\right)^{\prime}\right] .
\end{gathered}
$$

We multiply, again, each term in the $m$ summation of (21) by $1=-v W\left[I_{m}(v), K_{m}(v)\right]$, and turn the initial expression into a linear combination of integrals with summations of products of four Bessel functions. That linear combination yields an identically null result — one that is zero for any $s$ value - by virtue of the symmetries observed in (19) under interchange of different Bessel function types (see also comment after Eqs. (80) in $[5])$. As a result,

$$
\mathcal{E}_{C 1}^{1}(s)=0 .
$$

Equation (21) admits the following reinterpretation. Taking into account the fact that $I_{m}, K_{m}$ satisfy the modified Bessel equation, we apply partial integration to (21) omitting a 'boundary term' which vanishes for a given $s$ range that does not include $s=-1$ yet. Doing so, we find

$$
\begin{gathered}
\mathcal{E}_{C 1}^{1}(s)=-\frac{\hbar}{2} \frac{s a^{s-1}}{2 \pi^{2}} \mathrm{~B}\left(\frac{1}{2}, 1-\frac{s}{2}\right) \sin \left(\pi \frac{s}{2}\right) \\
\times\left[\int_{0}^{\infty} d v v^{1-s} \sum_{m=-\infty}^{\infty}\left(I_{m}(v) K_{m}(v)\right)^{\prime}+\frac{2}{1-s} \int_{0}^{\infty} d v v^{2-s} \sum_{m=-\infty}^{\infty} I_{m}(v) K_{m}(v)\right] .
\end{gathered}
$$

These integrals cannot be straightforwardly taken at $s=-1$ but, if this is ignored, we may formally put $s=-1$ and get

$\mathcal{E}_{C 1}^{1}(-1) \rightarrow-\frac{\hbar}{8 \pi a^{2}} \int_{0}^{\infty} d v v^{2} \sum_{m=-\infty}^{\infty}\left(I_{m}(v) K_{m}(v)\right)^{\prime}-\frac{\hbar}{8 \pi a^{2}} \int_{0}^{\infty} d v v^{3} \sum_{m=-\infty}^{\infty} I_{m}(v) K_{m}(v) .(24$

The first part could arguably be dismissed as a mere contact term because, from (18), it may be shown that it is local in $v$ (In fact it is possible to obtain $\left.\lim _{\phi \rightarrow 0} \sum_{m=-\infty}^{\infty}\left(I_{m}(v) K_{m}(v)\right)^{\prime} e^{i m \phi}=-\frac{1}{v}\right)$. The second part of (24) cancels the bulk contribution found in [5] (See formulas (72), (78) there and recall that the Casimir radial pressure is $P_{C}=\frac{1}{\pi a^{2}} \mathcal{E}_{C}$.)

Viewed in a different way, by the arguments in [13] (and references therein) all linear terms in $\left(\varepsilon_{2}-\varepsilon_{1}\right)$ have to be removed because they are the self-energy of the electromagnetic field due to polarizable particles. By that rule, one simply must take 
out the linear part, regardless of its particular form. This is actually a re-statement of the physical reason for the removal of the bulk contribution.

When going on to second order in $(\varepsilon-1)$, we take first the piece called $\mathcal{E}_{C 2}^{20 A}(s)$, as its calculation is most similar to that of $\mathcal{E}_{C 1}^{0}(s), \mathcal{E}_{C 1}^{1}(s)$. From the $\mathcal{E}_{C 2}^{20 A}(s)$ given in (15), the $L_{m 2}^{20 A}(y)$ in (9), expressions (10) with $y=i v$, introducing, once more, $1=-v W\left[I_{m}(v), K_{m}(v)\right]$, and using the same reasoning that led to (22), one gets

$$
\mathcal{E}_{C 2}^{20 A}(s)=0 .
$$

Now, selecting the lines in (15), which determine $\mathcal{E}_{C 2}^{00}(s), \mathcal{E}_{C 2}^{10}(s), \mathcal{E}_{C 2}^{20 B}(s), \mathcal{E}_{C 2}^{11}(s)$, the parts of (9) which define $L_{m 2}^{00}(y), L_{m 2}^{10}(y), L_{m 2}^{20 B}(y), L_{m 2}^{11}(y)$, the form of $\Delta_{m}^{(1,0)}(y)$ dictated by (10) (its square for the case of $L_{m 2}^{20 B}(y)$ ), and setting $y=i v$, we come to

$$
\begin{aligned}
& \mathcal{E}_{C 2}^{00}(s)= \frac{\hbar}{2} \frac{s a^{s-1}}{4 \pi^{2}} \mathrm{~B}\left(\frac{1}{2},-\frac{s}{2}\right) \sin \left(-\pi \frac{s}{2}\right) \int_{0}^{\infty} d v v^{2-s} \sum_{m=-\infty}^{\infty} I_{m}^{\prime 2}(v) K_{m}^{2}(v), \\
& \mathcal{E}_{C 2}^{10}(s)=\frac{\hbar}{2} \frac{s a^{s-1}}{4 \pi_{\infty}^{2}} \mathrm{~B}\left(\frac{1}{2}, 1-\frac{s}{2}\right) \sin \left(\pi \frac{s}{2}\right) \int_{0}^{\infty} d v v^{2-s} \quad \times \sum_{m=-\infty}\left[2 I_{m}(v) I_{m}^{\prime}(v) K_{m}(v) K_{m}^{\prime}(v)+v I_{m}^{\prime 2}(v) K_{m}(v) K_{m}^{\prime}(v)\right. \\
&\left.-\left(v+\frac{m^{2}}{v}\right) I_{m}^{2}(v) K_{m}(v) K_{m}^{\prime}(v)\right], \\
& \mathcal{E}_{C 2}^{20 B}(s)=\frac{\hbar}{2} \frac{s a^{s-1}}{8 \pi^{2}} \mathrm{~B}\left(\frac{1}{2}, 2-\frac{s}{2}\right) \sin \left(-\pi \frac{s}{2}\right) \int_{0}^{\infty} d v v^{2-s} \quad \times \sum_{m=-\infty} \quad\left[I_{m}^{\prime 2}(v) K_{m}^{2}(v)+I_{m}^{2}(v) K_{m}^{\prime 2}(v)\right. \\
&+2\left(1-v^{2}-m^{2}\right) I_{m}(v) I_{m}^{\prime}(v) K_{m}(v) K_{m}^{\prime}(v) \\
&+v^{2} I_{m}^{\prime 2}(v) K_{m}^{\prime 2}(v)+\left(v^{2}+2 m^{2}+\frac{m^{4}}{v^{2}}\right) I_{m}^{2}(v) K_{m}^{2}(v) \\
&+2 v\left(I_{m}^{\prime 2}(v) K_{m}(v) K_{m}^{\prime}(v)+I_{m}(v) I_{m}^{\prime}(v) K_{m}^{\prime 2}(v)\right) \\
&\left.\quad-2\left(v+\frac{m^{2}}{v}\right)\left(I_{m}(v) I_{m}^{\prime}(v) K_{m}^{2}(v)+I_{m}^{2}(v) K_{m}(v) K_{m}^{\prime}(v)\right)\right], \\
& \mathcal{E}_{C 2}^{11}(s)=\frac{\hbar}{2} \frac{s a^{s-1}}{2 \pi^{2}} \mathrm{~B}\left(\frac{3}{2}, 1-\frac{s}{2}\right) \sin \left(\pi \frac{s}{2}\right) \int_{0}^{\infty} d v v^{-s} \sum_{m=-\infty}^{\infty} m^{2} I_{m}^{2}(v) K_{m}^{2}(v) .
\end{aligned}
$$

The outcome of replacing the results (19) into (26) and expanding in $(s+1)$ is:

$$
\mathcal{E}_{C 2}^{00}(s)+\mathcal{E}_{C 2}^{10}(s)+\mathcal{E}_{C 2}^{20 B}(s)+\mathcal{E}_{C 2}^{11}(s)=\frac{\hbar}{a^{2}} \widehat{\mathcal{E}}(s+1)+\mathcal{O}\left((s+1)^{2}\right),
$$

with $\widehat{\mathcal{E}}=\frac{23}{5760 \pi}$. Formulas $(25)$ and $(27)$ make evident that

$$
\lim _{s \rightarrow-1} \mathcal{E}_{C 2}(s)=0,
$$

i.e., the $(\varepsilon-1)^{2}$ contribution to the Casimir energy per unit length in the dilute-dielectric approximation is zero, as we wished to prove.

Employing a regularization which analytically continues the vacuum energy as a function of the eigenmode power, we have found a pure Casimir term (in the sense 
of [2]) that is seen to vanish to the order of $(\varepsilon-1)^{2}$. Remarkably, for the analogous problem with light velocity conservation condition $[1,12]$ the result is null to the order

of $\xi^{2} \equiv\left(\frac{\varepsilon_{1}-\varepsilon_{2}}{\varepsilon_{1}+\varepsilon_{2}}\right)^{2}$. In fact, we have applied a form of zeta function regularization, whose links to other techniques have been studied in e.g. [14]. The sight of (19) makes us evoke the words of [15] and proclaim that a forest of gamma functions has grown out of an analytic continuation.

A divergence at third order in $(\varepsilon-1)$ introduces an unavoidable ambiguity [4] (for further discussions on divergences see [16].) No universal agreement exists on the physical interpretation of the technique used, as commented in [15]. The nature of a third order divergence, viewed as a weak-coupling limit, has been considered in [17].

\section{Acknowledgments}

A.R. thanks V.V. Nesterenko, M. Bordag and I.G. Pirozhenko for observations and comments. The authors acknowledge useful conversations with Inés Cavero-Peláez. The work of K.A.M. is supported in part by a grant from the U.S. Department of Energy.

\section{References}

[1] Milton KA, Nesterenko AV and Nesterenko VV 1999 Phys. Rev. D 59105009 (Preprint hepth/9711168 v3. The v1 version did not include the first author)

[2] Barton G 2001 J. Phys. A 344083

[3] Milton KA and Ng YJ 1997 Phys. Rev. E 55 4207; Brevik IH, Marachevsky VN and Milton KA 1999 Phys. Rev. Lett. 82 3948; Barton G 1999 J. Phys. A 32525

[4] Bordag M and Pirozhenko IG 2001 Phys. Rev. D 64025019 (Preprint hep-th/0102193)

[5] Cavero-Peláez I and Milton KA 2005 Ann. Phys. NY 320108 (Preprint hep-th/0412135 v2)

[6] Romeo A and Milton KA 2005 Phys. Lett. B 621309 (Preprint hep-th/0504207)

[7] Stratton JA 1941 Electromagnetic Theory (McGraw-Hill)

[8] van Kampen NG, Nijboer BRA and Schram K 1968 Phys. Lett. A 26307

[9] Milton KA, DeRaad LL and Schwinger JS 1978 Ann. Phys. NY 115388

[10] Brevik I, Jensen B and Milton KA 2001 Phys. Rev. D 64088701 (Preprint hep-th/0004041)

[11] Gradshteyn IS and Ryzhik IM 1994 Table of Integrals, Series and Products, Fifth Ed. (Acad. Press)

[12] Klich I 2000 Phys. Rev. D 61025004 (Preprint hep-th/9908101); Klich I and Romeo A 2000 Phys. Lett. B 476369 (Preprint hep-th/9912223)

[13] Lambiase G, Scarpetta G and Nesterenko VV 2001 Mod. Phys. Lett. A 161983 (Preprint hepth/9912176)

[14] Cognola G, Vanzo L and Zerbini S 1992 J. Math. Phys. 33 222; Beneventano CG and Santangelo EM 1996 Int. J. Mod. Phys. A 112871 (Preprint hep-th/9501122)

[15] Fulling SA 2003 J. Phys. A 366857 (Preprint quant-ph/0302117)

[16] Graham N, Jaffe RL, Khemani V, Quandt M, Schroeder O and Weigel H 2004 Nucl. Phys. B 677379 (Preprint hep-th/0309130) and references therein

[17] Cavero-Peláez I, Milton KA and Wagner J 2005 Preprint hep-th/0508001 v2 